\title{
A TOPOLOGICAL TRANSLATION OF A FUNDAMENTAL PROBLEM IN CLUSTER SET THEORY
}

\author{
J. A. EIDSWICK
}

\begin{abstract}
Two conjectures related to the problem of finding a "large" family of approach curves along which cluster sets can be preassigned are shown to be equivalent to special cases of the normal Moore space conjecture.
\end{abstract}

1. In [4] the author posed the following problems for real-valued functions $f$ of two real variables. (Here "approach curves" approach the origin and $C(f, \gamma)$ denotes the cluster set of $f$ at 0 along the curve $\left.\gamma_{\bullet}\right)$

I. Find a "small" subset $\Gamma_{0}$ of the set $\Gamma$ of all approach curves such that for each function $f,\left\{C\left(f, \gamma_{0}\right): \gamma_{0} \in \Gamma_{0}\right\}$ determines $C(f, \gamma)$ for all $\gamma$ in $\Gamma$.

II. Find a "large" subset $\Gamma_{0}$ of $\Gamma$ such that to any collection $\left\{C\left(\gamma_{0}\right): \gamma_{0} \in \Gamma_{0}\right\}$ of closed sets there corresponds a function $f$ such that $C\left(f, \gamma_{0}\right)=C\left(\gamma_{0}\right)$ for all $\gamma_{0}$ in $\Gamma_{0}$.

A satisfactory solution of Problem I was found in [4] by generalizing Rosenthal's "Approach Curve Theorem", but Problem II was only partially solved. It was conjectured that "A family $\Gamma_{0}$ is a solution of Problem II if and only if $\Gamma_{0}$ has a nonintersecting truncation". Put another way, the conjecture is that a family $\Gamma_{0}$ satisfies the condition of Problem II if and only if it is somehow possible to "clip" the curves of $\Gamma_{0}$ in such a way that the resulting family of "stubs" (minus the origin) is nonintersecting.

In this paper it is shown that the above conjecture is equivalent to the normal Moore space conjecture for a certain family of spaces. In these spaces, collectionwise Hausdorff implies collectionwise normality. Consequently the conjecture is consistent with the usual axioms of set theory. Conjecture (1) of [4] is similarly translated and its consistency established.

For the definition of a Moore space and the general status of the Moore space conjecture, see [11], [5] - [7], [12]-[14]; for the theory of cluster sets, see [3] and [8].

2. For a family $\Gamma_{0}$ of approach curves, let $X\left(\Gamma_{0}\right)$ consist of (a) the points of the punctured plane $R^{2}-\{0\}$ and (b) the curves of $\Gamma_{0}$ (thought of

Received by the editors September 7, 1974.

AMS (MOS) subject classifications (1970). Primary 26A15, 54D15, 54E30, 54E35; Secondary 02K05, 04A30.

Key words and phrases. Cluster sets, approach curves, truncations, Moore spaces, picket fence spaces, metrizability. 
as copies of the origin). Put the discrete topology on $\mathbf{R}^{2}-\{0\}$ and take as a neighborhood basis of a point $\gamma_{0}$ of $\Gamma_{0}$ the truncations of $\gamma_{0}$ (regarded as point-sets in $\mathbf{R}^{2}$, the origin being replaced by the point $\gamma_{0}$ ). The topological space generated is clearly $T_{1}$ and developable.

As in [4], a family $\Gamma_{0}$ is called comparable if every pair of curves from $\Gamma_{0}$ has a nonintersecting truncation. Recall that a necessary condition for $\Gamma_{0}$ to be a solution of Problem II is that it be comparable.

Theorem 1. For any family $\Gamma_{0}$ of approach curves,

(i) $X\left(\Gamma_{0}\right)$ is a Moore space if and only if $\Gamma_{0}$ is comparable,

(ii) $X\left(\Gamma_{0}\right)$ is normal if and only if $\Gamma_{0}$ is a solution of Problem II, and

(iii) $X\left(\Gamma_{0}\right)$ is collectionwise normal if and only if $\Gamma_{0}$ has a nonintersecting truncation.

Corollary 1. The following assertions are equivalent:

(T) A family $\Gamma_{0}$ is a solution of Problem II if and only if $\Gamma_{0}$ has a nonintersecting truncation.

(M) Every normal Moore space $X\left(\Gamma_{0}\right)$ is metrizable.

Corollary 2. Assertion $(\mathrm{T})$ is consistent with the usual axioms of set theory.

By the usual axioms is meant the Zermelo-Fraenkel axioms together with the axiom of choice (ZFC).

Proof of (i). Clearly, a family $\Gamma_{0}$ is comparable if and only if the space $X\left(\Gamma_{0}\right)$ is Hausdorff. Also, if $\Gamma_{0}$ is comparable, then basic neighborhoods of points of type (b) are closed, and it follows that $X\left(\Gamma_{0}\right)$ is regular.

Proof of (ii). If $\Gamma_{0}$ is a solution of Problem II, then any two disjoint subsets of $\Gamma_{0}$ can be separated by disjoint open sets. To see this, let $\Gamma_{1}$ and $\Gamma_{2}$ be disjoint subsets of $\Gamma_{0}$ and let $f$ be a function such that $C(f, \gamma)=\{0\}$ or $\{1\}$ according as $\gamma$ is in $\Gamma_{1}$ or $\Gamma_{2}$. By definition of cluster set, there exist truncations $T_{1}$ and $T_{2}$ of $\Gamma_{1}$ and $\Gamma_{2}$, respectively, such that $f$ is less than $1 / 2$ on $T_{1}$ and greater than $1 / 2$ on $T_{2}$. The desired separation is given by: $U_{1}=T_{1} \cup \Gamma_{1}, U_{2}=T_{2} \cup \Gamma_{2}$. To prove normality of $X\left(\Gamma_{0}\right)$, let $C_{1}$ and $C_{2}$ be disjoint closed subsets and put $\Gamma_{1}=C_{1} \cap \Gamma_{0}$, $\Gamma_{2}=C_{2} \cap \Gamma_{0}, D_{1}=C_{1}-\Gamma_{1}, D_{2}=C_{2}-\Gamma_{2}$. Then it is easy to see that a separation is given by: $\left(U_{1}-\bar{D}_{2}\right) \cup D_{1},\left(U_{2}-\bar{D}_{1}\right) \cup D_{2}$.

The converse will be proved in stages. First, consider the case where $C\left(\gamma_{0}\right)$ is either $\{0\}$ or $\{1\}$ for every $\gamma_{0} \in \Gamma_{0}$. In this case, normality of $X\left(\Gamma_{0}\right)$ implies that $\Gamma_{1}=\left\{\gamma_{0} \in \Gamma_{0}: C\left(\gamma_{0}\right)=\{0\}\right\}$ and $\Gamma_{2}=\left\{\gamma_{0} \in \Gamma_{0}: C\left(\gamma_{0}\right)=\right.$ $\{1\}\}$ can be separated by disjoint open sets $U_{1}$ and $U_{2}$, and we need only define $f$ so that $f\left(U_{1}-\Gamma_{1}\right)=0$ and $f\left(U_{2}-\Gamma_{2}\right)=1$.

Next suppose every $C\left(\gamma_{0}\right)$ consists of a single point $c\left(\gamma_{0}\right)=\Sigma_{1}^{\infty}{ }^{c}{ }_{n}\left(\gamma_{0}\right) / 2^{n}$ where each $c_{n}\left(\gamma_{0}\right)$ is either 0 or 1 . For each $n$, let $f_{n}$ be a function on 
$\mathbf{R}^{2}$ with range $\{0,1\}$ such that $C\left(f_{n}, \gamma_{0}\right)=\left\{c_{n}\left(\gamma_{0}\right)\right\}$ for every $\gamma_{0} \in \Gamma_{0}$, and define $f(p)=\Sigma_{1}^{\infty} f_{n}(p) / 2^{n}$ for $p \in \mathbf{R}^{2}$. Then for each $\gamma_{0}, \lim f(p)=c\left(\gamma_{0}\right)$ as $p \rightarrow 0$ along $\gamma_{0}$; i.e., $C\left(f, \gamma_{0}\right)=C\left(\gamma_{0}\right)$ for every $\gamma_{0} \in \Gamma_{0}$. More generally, if each $C\left(\gamma_{0}\right)$ consists of a single point in $[-\infty, \infty]$, let $\phi$ be any homeomorphism from $[-\infty, \infty]$ onto $[0,1]$ and let $g$ be a function on $\mathbf{R}^{2}$ such that $C\left(g, \gamma_{0}\right)=\phi\left(C\left(\gamma_{0}\right)\right)$ for every $\gamma_{0} \in \Gamma_{0}$. Then $C\left(\phi^{-1} g, \gamma_{0}\right)=C\left(\gamma_{0}\right)$ for every $\gamma_{0} \in \Gamma_{0}$.

The following modification of Bettazzi's construction (cf. [8, p. 319]) completes the proof of (ii): For $\gamma_{0} \in \Gamma_{0}$, let $\left\{a_{1}\left(\gamma_{0}\right), a_{2}\left(\gamma_{0}\right), \ldots\right\}$ be a countable dense subset of $C\left(\gamma_{0}\right)$ and for each $m$, let $f_{m}$ be a function such that $C\left(f_{m}, \gamma_{0}\right)=\left\{a_{m}\left(\gamma_{0}\right)\right\}$ for every $\gamma_{0} \in \Gamma_{0}$. Next, let the sequence of annuli $A_{n}=\{p: 1 /(n+1)<|p| \leq 1 / n\}, n=1,2, \ldots$, be partitioned into a denumerable number of subsequences. Finally, define $f(p)=f_{m}(p)$ on the annuli from the $m$ th subsequence to get $C\left(f, \gamma_{0}\right)=C\left(\gamma_{0}\right)$ for every $\gamma_{0} \in \Gamma_{0}$.

Proof of (iii). If $X\left(\Gamma_{0}\right)$ is collectionwise normal, then the discrete subspace $\Gamma_{0}$ can be separated and hence $\Gamma_{0}$ has a nonintersecting truncation. Conversely, let $\left\{C_{\alpha}: \alpha \in A\right\}$ be a discrete collection of closed sets and let $\Gamma_{a}=C_{a} \cap \Gamma_{0}, D_{a}=C_{a}-\Gamma_{a}$. If $T$ is a nonintersecting truncation of $\Gamma_{0}$, let $T_{a}, a \in A$, be the restriction of $T$ to $\Gamma_{a}$. Then the open sets $\left(\left(T_{a} \cup \Gamma_{\alpha}\right)-\overline{U_{\xi \neq a} D_{\xi}}\right) \cup D_{\alpha}$, separate the sets $C_{\alpha}$.

Proof of Corollary 1. By a theorem of Bing [1], metrizability is equivalent to collectionwise normality for Moore spaces. Hence, both statements are equivalent to "normality implies collectionwise normality" for the spaces $X\left(\Gamma_{0}\right)$.

Proof of Corollary 2. Follows from [6].

We turn now to the more basic nonintersecting truncation problem of [4] and its translation. For a set $S$ of real numbers and a real-valued function $m$ defined on $S$, let $(S, m)$ denote the family of half-lines $y=m(s) x$ $+s, x>0, s \in S$. It is easy to see that a family $(S, m)$ has a nonintersecting truncation if and only if

(a) There exists a positive function $p$ on $S$ such that

$$
(m(s)-m(t)) /(t-s)<\max (p(s), p(t))
$$

whenever $s, t, \in S, s \neq t$. Conjecture (1) of [4] is that $(\alpha)$ is equivalent to

$(\beta)$ For each decomposition $(A, B)$ of $S$ there exists a positive function $p$ on $S$ such that

$$
(m(a)-m(b)) /(b-a)<\max (p(a), p(b))
$$

whenever $a \in A, b \in B$.

The equivalence question resembles Bing's question "(b) implies (a)" of [2]. Indeed, it was this resemblance that led Smith [10] in a proof of the consistency of " $(\beta)$ implies $(\alpha)$ ". A more direct proof is given here.

To each family $(S, m)$ associate a "picket fence space" $X(S, m)$ as 
follows: The points of $X(S, m)$ are the points of the right half-plane $x>0$ together with the points of $S$, the right half-plane is given the discrete topology, and basic neighborhoods of points $s$ of $S$ are the truncated lines $y=$ $m(s) x+s, 0 \leq x<\tau, \tau>0$.

Theorem 2. For any family $(S, m)$,

(i) $X(S, m)$ is a Moore space,

(ii) $X(S, m)$ is normal if and only if $(\beta)$, and

(iii) $X(S, m)$ is collectionwise normal if and only if $(\alpha)$.

Corollary 1. The following assertions are equivalent:

(T) $(\alpha)$ is equivalent to $(\beta)$.

(M) Every normal Moore space $X(S, m)$ is metrizable.

Corollary 2. Assertion ( $\mathrm{T})$ is consistent with the usual axioms of set theory.

Proofs. It is easy to see that $(\beta)$ holds if and only if for each decomposition $(A, B)$ of $S$, there exists a truncation of $(S, m)$ such that no truncated line from $(A, m)$ intersects a truncated line from $(B, m)$. Thus, in the terminology of [14], $(\alpha)$ holds if and only if $S$ is separated and $(\beta)$ holds if and only if $S$ is normalized. Parts (ii) and (iii) now follow as in the proof of Theorem 1.

Part (i) is obvious and the corollaries follow as in Theorem 1.

Remark. It can be shown that if $(S, m)$ has a nonintersecting truncation for every $m$, then every subset of $S$ is relatively $F_{\sigma}$. This implies that conjecture (2) of [4] is false. Conjecture (3) of [4] is also false.

Question. Does there exist a nonmetrizable normal picket fence space? In particular, if $S$ is a $Q$-set (an uncountable set every subset of which is relatively $\left.F_{\sigma}\right)$, is it possible to find $m$ such that $(S, m)$ has no nonintersecting truncation?

\section{REFERENCES}

1. R. H. Bing, Metrization of topological spaces, Canad. J. Math. 3 (1951), $175-186$

2. - A translation of the normal Moore space conjecture, Proc. Amer. Math. Soc. 16 (1965), 612-619. MR 31 \#6201.

3. E. F. Collingwood and A. J. Lohwater, The theory of cluster sets, Cambridge Tracts in Math. and Math. Phys., no. 56, Cambridge Univ. Press, Cambridge, 1966, MR 38 \#325.

4. J. A. Eidswick, On some fundamental problems in cluster set theory, Proc. Amer. Math. Soc. 39 (1973), 163-168. MR 47 \#2008.

5. William Fleissner, Thesis, University of California, Berkeley, Calif., 1974.

6. - Normal Moore spaces in the constructible universe, Proc. Amer. Math. Soc. 46 (1974), 294-298.

7. — When is Jones' space normal? Proc. Amer. Math. Soc. (submitted).

8. E. W. Hobson, The theory of functions of a real variable. Vol. 1, 3rd ed., Dover, New York, 1957. 
9. Kiyoshi Noshiro, Cluster sets, Ergebnisse der Mathematik und ihrer Grenzgebiete, N.F., Heft 28, Springer-Verlag, Berlin, 1960. MR 24 \#A3295.

10. Neil G. Smith, On a set-theoretic offshoot of the normal Moore space question, Thesis, University of Nebraska, 1973.

11. L. A. Steen, Conjectures and counterexamples in metrization theory, Amer. Math. Monthly 79 (1972), 113-132. MR 46 \#8186.

12. F. D. Tall, Set-theoretic consistency results and topological theorems concerning the normal Moore space conjecture and related problems, Thesis, University of Wisconsin, Madison, Wis., 1969.

13. - New results on the normal Moore space problem, Proc. Washington State Univ. Conf. on General Topology (Pullman, Wash., 1970), Pi Mu Epsilon, Dept. of Math., Washington State Univ., Pullman, Wash., 1970, pp. 120-126. MR 41 \#9195.

14. - A set-theoretic proposition implying the metrizability of normal Moore spaces, Proc. Amer. Math. Soc. 33 (1972), 195-198. MR 45 \#9285.

DEPARTMENT OF MATHEMATICS, UNIVERSITY OF NEBRASKA, LINCOLN, NEBRASKA 68508 